Real Analysis Exchange

Vol. 24(2), 1998/9, pp. 765-780

T. H. Steele, Department of Mathematics, Weber State University, Ogden, UT 84408-1702; e-mail: thsteele@weber.edu

\title{
ITERATIVE STABILITY IN THE CLASS OF CONTINUOUS FUNCTIONS
}

\begin{abstract}
Let $\mathcal{K}$ be the class of compact subsets of $I=[0,1]$, and $\mathcal{K}^{*}$ consist of the nonempty closed subsets of $\mathcal{K}$. We study the maps $\Lambda: C(I, I) \rightarrow \mathcal{K}$ and $\Omega: C(I, I) \rightarrow \mathcal{K}^{*}$ defined so that $\Lambda(f)$ is the set of $\omega$-limit points of $f$, and $\Omega(f)$ is the collection of $\omega$-limit sets of $f$. We find that, in general, neither map is continuous. We do get more positive results, however, if we restrict ourselves to certain types of $\omega$-limit sets and better behaved classes of functions. We find that when $f$ has only a finite number of $\omega$-limit sets, each demonstrating a certain type of stability, the function $\Omega$ is continuous at $f$. The map $\Omega \mid \mathcal{E}$ is also studied, where $\mathcal{E}$ is composed of those continuous functions with zero topological entropy, and a significant degree of stability of $\Omega \mid \mathcal{E}$ is established.
\end{abstract}

\section{Introduction}

The iterative behavior of continuous self-maps of a compact interval has received considerable attention in recent years. We will concern ourselves with some related questions concerning stability within the family of continuous self-maps of the interval $I=[0,1]$. We begin with a rather general query. If $f$ and $g$, both continuous self-maps of $I$, are close to one another, are their iterative characteristics in some way similar?

If by similar we mean that the functions are topologically conjugate to one another, then we can never achieve a positive result in the space $\{C(I, I),\|\cdot\|\}$, where we endow the set of continuous functions mapping $I$ to $I$ with the supremum metric. Since any $f$ in $C(I, I)$ must have a fixed point, for any $\epsilon>0$ we can find $g$ in $C(I, I)$ so that $\|f-g\|<\epsilon$, yet $g$ has considerably different dynamics than $f$ on a neighborhood of the fixed point of $f$. In fact, we can take $g$ to equal $f$ outside of our neighborhood of the fixed point, and define $g$ however we choose on that neighborhood so long as $g$ is continuous.

Mathematical Reviews subject classification: 26A18

Received by the editors November 15, 1996 
Near any fixed point of $f$, then, arbitrarily small perturbations can change the local dynamics considerably. It is still possible, however, that if $f$ is perturbed only slightly to obtain $g$, then $g$ will have $\omega$-limit sets that are at least close to those of $f$. For example, if we let $f \equiv 0$ and take $g$ so that $\|g-f\|<\epsilon$, then all the $\omega$-limit sets of $g$ are contained in $[0, \epsilon]$, and hence in some sense close to $f$ 's unique $\omega$-limit set $\{0\}$. We will pursue this idea at some length by making use of the Hausdorff metric $\mathcal{H}$ on the class of closed sets $\mathcal{K}$ contained in $[0,1]$. In our above example, the $\omega$-limit sets of $g$ and $f$ would be $\epsilon$-close to one another since every point in one is within $\epsilon$ of a point in the other.

We proceed through several sections.

In section 2 we present the notation and definitions we will use throughout the balance of the paper. We also recall some important, previously known results.

In section 3 we develop examples which show that the best possible result is not valid; in general, the $\omega$-limit points of $f$ and $g$ need not be close to one another regardless of how close $f$ and $g$ are to each other. Nevertheless, these examples help us narrow our focus to the more positive results found in the ensuing sections.

In section 4 we focus our attention on convergent sequences $\left\{f_{n}\right\}$ in $C(I, I)$ that possess convergent $\omega$-limit sets $\omega_{n}$, and find that for functions of zero topological entropy, we are able to assert quite a bit about the relationship between $f=\lim _{n \rightarrow \infty} f_{n}$ and $\omega=\lim _{n \rightarrow \infty} \omega_{n}$. In this setting, the maximal perfect subset of $\omega$ is always an $\omega$-limit set of $f$.

In section 5 we show that if $f$ has only a finite number of $\omega$-limit sets, and $g$ is sufficiently close to $f$, then the $\omega$-limit sets of $g$ are close to those of $f$, provided that $f$ 's $\omega$-limit sets themselves meet a certain stability criterion.

In section 6 we conclude with some open problems and a few observations.

\section{Preliminaries}

We shall be concerned with the class $C(I, I)$ of continuous functions mapping the unit interval $I=[0,1]$ to itself, and the iterative properties this class of functions possesses. For $f$ in $C(I, I)$ and any integer $n \geq 1, f^{n}$ denotes the $n^{\text {th }}$ iterate of $f$. For $x$ in $I$, we call the set of all subsequential limits of the sequence $\left\{f^{n}(x)\right\}_{n=0}^{\infty}$ the $\omega$-limit set of $f$ generated by $x$, and write $\omega(x, f)$. We let $\Lambda(f)=\cup_{x \in I} \omega(x, f)$ represent the $\omega$-limit points of $f$, while $\Omega(f)=\{\omega(x, f): x \in I\}$ denotes the set composed of the $\omega$-limit sets of $f$. To a very large extent, our study is one of the stability of $\Lambda(f)$ and $\Omega(f)$ as $f$ undergoes perturbations.

We will be working primarily in four metric spaces. We will use the regular, 
Euclidean metric $d$ on $I=[0,1]$, and make occasional use of neighborhoods of closed sets $F$ of the form $B_{\epsilon}(F)=\{x \in I: d(x, y)<\epsilon, y \in F\}$. Within $C(I, I)$ we will use the supremum metric given by $\|f-g\|=\sup \{|f(x)-g(x)|: x \in I\}$. Our third metric space $\{\mathcal{K}, \mathcal{H}\}$ is composed of the class of nonempty closed sets $\mathcal{K}$ in $I$ endowed with the Hausdorff metric $\mathcal{H}$ given by $\mathcal{H}(E, F)=\inf \{\delta>$ $\left.0: E \subset B_{\delta}(F), F \subset B_{\delta}(E)\right\}$. This space is compact [4]. Our final metric space $\left\{\mathcal{K}^{*}, \mathcal{H}^{*}\right\}$ consists of nonempty closed subsets of $\mathcal{K}$. Thus, $K \in \mathcal{K}^{*}$ if $K$ is a nonempty family of nonempty closed sets in $I$ such that $K$ is closed in $\mathcal{K}$ with respect to $\mathcal{H}$. We endow $\mathcal{K}^{*}$ with the metric $\mathcal{H}^{*}$, so that $K_{1}$ and $K_{2}$ are close with respect to $\mathcal{H}^{*}$ if each member of $K_{1}$ is close to some member of $K_{2}$ with respect to $\mathcal{H}$, and vice versa.

Our interest in, and the utility of, the metric spaces $\{\mathcal{K}, \mathcal{H}\}$ and $\left\{\mathcal{K}^{*}, \mathcal{H}^{*}\right\}$ stems from the following two theorems from [1] and [2], respectively.

Theorem 2.1. For any $f$ in $C(I, I)$, the set $\Lambda(f)$ is closed in $I$.

Theorem 2.2. For any $f$ in $C(I, I)$, the set $\Omega(f)$ is closed in $\{\mathcal{K}, \mathcal{H}\}$.

To a large extent, then, our stability queries can be formulated via the maps $\Lambda:\{C(I, I),\|\|.\} \rightarrow\{\mathcal{K}, \mathcal{H}\}$ given by $f \rightarrow \Lambda(f)$ and $\Omega:\{C(I, I),\|\|.\} \rightarrow$ $\left\{\mathcal{K}^{*}, \mathcal{H}^{*}\right\}$ given by $f \rightarrow \Omega(f)$.

In much of section 4 we will restrict our attention to a closed subset of $C(I, I)$ composed of those functions $f$ having zero topological entropy, denoted by $\mathbf{h}(f)=0$. The reader is referred to Theorem A of [9] for an extensive listing of equivalent formulations of topological entropy zero. For our purposes, it suffices to note that every periodic orbit of a continuous function with zero topological entropy has cardinality a power of two. The following theorem, due to Smital[10], sheds considerable light on the structure of infinite $\omega$-limit sets for functions with zero topological entropy.

Theorem 2.3. If $\omega$ is an infinite $\omega$-limit set for $f$ in $C(I, I)$ possessing zero topological entropy, then there exists a sequence of closed intervals $\left\{J_{k}\right\}_{k=1}^{\infty}$ in [0,1] such that

- for each $k,\left\{f^{i}\left(J_{k}\right)\right\}_{i=1}^{2^{k}}$ are pairwise disjoint and $J_{k}=f^{2^{k}}\left(J_{k}\right)$.

- for each $k, J_{k+1} \cup f^{2^{k}}\left(J_{k+1}\right) \subseteq J_{k}$.

- for each $k, \omega \subseteq \cup_{i=1}^{2^{k}} f^{i}\left(J_{k}\right)$.

- for each $k$ and $i, \omega \cap f^{i}\left(J_{k}\right) \neq \emptyset$. 
We make the following definitions with Smital's Theorem in mind. Let $\omega$ be an infinite compact subset of $I$, and let $f$ map $\omega$ into itself. We call $f$ a simple map on $\omega$ if $\omega$ has a decomposition $S \cup T$ into compact portions that $f$ exchanges, and $f^{2}$ is simple on each of these portions. From Smital's Theorem one sees that every map $f$ with zero topological entropy is simple on each of its infinite $\omega$-limit sets $\omega=\omega(x, f)$. Let $\left\{J_{k}\right\}_{k=1}^{\infty}$ be a nested sequence of compact periodic intervals of $\omega$ and $f$ as described in Smital's Theorem. Every set of the form $\omega \cap f^{i}\left(J_{n}\right)$ is periodic of period $2^{n}$, and we call each such set a periodic portion of rank $n$. This system of periodic portions of $\omega$, or of the corresponding periodic intervals, is called the simple system of $\omega$ with respect to $f$.

We will need the following notation in section 5 when we study the function $\Omega:\{C(I, I),\|\|.\} \rightarrow\left\{\mathcal{K}^{*}, \mathcal{H}^{*}\right\}$ at all points $f$ for which $\Omega(f)$ is finite. Suppose $\omega \in \Omega(f)$, and $\omega=\omega(x, f)$ contains only finitely many points, say $|\omega|=n$. We say that $\omega$ is a stable $\omega$-limit set of $f$ if $f^{n}(x)-x$ is not unisigned in any deleted neighborhood of $x$.

\section{Examples}

This section will be dedicated primarily to the development of examples. These examples will provide us with some insight into the behavior of our functions $\Lambda$ and $\Omega$ as well as focus our efforts in the ensuing sections.

Example 1. Consider $f_{n}(x)=x^{\frac{n-1}{n}}$. As $n$ goes to infinity, we see that $f_{n}$ goes to the identity function $f$. Thus, $\Lambda(f)=[0,1]$. Since $\Lambda\left(f_{n}\right)=\{0,1\}$ for all $n$, we see that $\Lambda$ is not continuous at $f$, so that $\Omega$ must necessarily be discontinuous there, too. While this does rule out the best possible result - that $\Omega$, and therefore $\Lambda$, are continuous - our example does not rule out a natural generalization of the theorem found in [2].

Recall that our four authors in [2] show that if $\left\{\omega_{n}\right\} \subseteq \Omega(f)$, and $\omega_{n} \rightarrow \omega$ in $K$, then $\omega \in \Omega(f)$. In example $1,\{0\} \in \Omega\left(f_{n}\right)$ for every $n$, and $\{0\} \in \Omega(f)$. Perhaps, then, the following is true: If $\omega_{n} \in \Omega\left(f_{n}\right)$ for each $n, f_{n} \rightarrow f$ and $\omega_{n} \rightarrow \omega$, then $\omega \in \Omega(f)$. This conjecture simplifies to the result of [2] if we let $f_{n}=f$ for all $n$.

For our next example, we need the following definition. Let $M$ be a nowhere dense compact set in $I$, with $A=\left\{a_{0}, a_{1}, \ldots, a_{k-1}\right\} \neq \emptyset$ a set of limit points of $M$. Suppose there is a system $\left\{M_{n}^{i}\right\}_{n=0}^{\infty}, i=0,1, \ldots, k-1$ of nonempty pairwise disjoint compact subsets of $M$ such that $M \backslash \cup_{i, n} M_{n}^{i}=A$ and $\lim _{n \rightarrow \infty} M_{n}^{i}=a_{i}$ for each $i$. Let $f: M \rightarrow M$ be a continuous map with $A$ a $k$-cycle of $f$ such that $f\left(a_{i}\right)=a_{i-1}$ for $i>0$ and $f\left(a_{0}\right)=a_{k-1}$. If $f\left(M_{n}^{i}\right)=M_{n}^{i-1}$ for $i>0$ and any $n, f\left(M_{n}^{0}\right)=M_{n-1}^{k-1}$ for $n>0$, and $f\left(M_{0}^{0}\right)=a_{k-1}$, then $M$ is called a 
homoclinic set of order $k$ with respect to $f$.

Example 2. We will construct a sequence of homoclinic $\omega$-limit sets $\omega_{n}$ for functions $f_{n}$ in $C(I, I)$ so that $\omega_{n} \rightarrow \omega, f_{n} \rightarrow f$, yet $\omega$ is not contained in $\Lambda(f)$. This negates our conjectured generalization of the result from [2].

We begin by constructing our $\omega$-limit sets $\omega_{n}$. For each portion $M_{n}^{i}$, we take a scaled copy of the middle thirds Cantor set with the indicated convex closure.

For $\omega_{1}$, let $a_{0}=\frac{1}{2}$ and $\overline{\operatorname{conv}} M_{n}^{0}=\left[\frac{1}{2}+\frac{1}{2^{2+n}}, \frac{1}{2}+\frac{1}{2^{2+n}}+\frac{1}{2^{3+n}}\right]$. Set $A_{0}=$ $a_{0} \cup\left\{\cup_{n=0}^{\infty} M_{n}^{0}\right\}$. Now, let $a_{1}=0$ and $\overline{\operatorname{conv}} M_{n}^{1}=\left[\frac{1}{2^{2+n}}, \frac{1}{2^{2+n}}+\frac{1}{2^{3+n}}\right]$.

For $\omega_{2}$, we begin with the set $A_{0}$ described above, and take $a_{1}=\frac{1}{4}$ and $\overline{\text { conv }} M_{n}^{1}=\left[\frac{1}{4}+\frac{1}{2^{3+n}}, \frac{1}{4}+\frac{1}{2^{3+n}}+\frac{1}{2^{4+n}}\right]$; let $A_{1}=a_{1} \cup\left\{\cup_{n=0}^{\infty} M_{n}^{1}\right\}$. Now, let $a_{2}=0$ and $\overline{\operatorname{conv}} M_{n}^{2}=\left[\frac{1}{2^{3+n}}, \frac{1}{2^{3+n}}+\frac{1}{2^{4+n}}\right]$.

In general, for $\omega_{m}$, we begin with the sets $A_{0}, A_{1}, \ldots, A_{m-2}$ and take $a_{m-1}=$ $\frac{1}{2^{m}}$ and $\overline{\operatorname{conv}} M_{n}^{m-1}=\left[\frac{1}{2^{m+1}}+\frac{1}{2^{m+2+n}}, \frac{1}{2^{m+1}}+\frac{1}{2^{m+2+n}}+\frac{1}{2^{m+3+n}}\right]$; let $A_{m-1}=$ $a_{m-1} \cup\left\{\cup_{n=0}^{\infty} M_{n}^{m-1}\right\}$. Now, let $a_{m}=0$ and $\overline{\operatorname{conv}} M_{n}^{m}=\left[\frac{1}{2^{m+2+n}}, \frac{1}{2^{m+2+n}}+\right.$ $\left.\frac{1}{2^{m+3+n}}\right]$.

We see that each of our sets $\omega_{n}$ will be homoclinic of order $n+1$, and the sequence $\left\{\omega_{n}\right\}$ converges in $\mathcal{K}$ to the set $\omega=\{0\} \cup\left\{\cup_{n=0}^{\infty} A_{n}\right\}$. How our functions $f_{n}: \omega_{n} \rightarrow \omega_{n}$ are defined is clear from our definition of a homoclinic trajectory as well as the construction of the sets $\omega_{n}$. Moreover, since each resulting $f_{n}$ is continuous, we can use [6] to extend $f_{n}: \omega_{n} \rightarrow \omega_{n}$ to a function we will also call $f_{n}$ that is in $C(I, I)$ and has the property that $\omega_{n}=\omega\left(x, f_{n}\right)$ for some $x \in I$. Since we can take $f_{n}\left|A_{1} \cup \ldots \cup A_{m}=f_{k}\right| A_{1} \cup \ldots \cup A_{m}$ for all $n$ and $k$ greater than $m+2$, and $A_{n} \rightarrow 0$ as $n \rightarrow \infty$, we can take our $f_{n}$ so that $f=\lim _{n \rightarrow \infty} f_{n}$ exists, and $f(x)=0$ for $x \in\left[\frac{1}{2}, 1\right]$. Thus, $\Lambda(f) \cap\left[\frac{1}{2}, 1\right]=\emptyset$ as $f(0)=\lim _{n \rightarrow \infty} f_{n}(0)=\lim _{n \rightarrow \infty} \frac{1}{2^{n}}$.

It is worth pointing out that not only is $\omega$ not an $\omega$-limit set of $f$, but we lose a considerable portion of our $\omega$-limit points as well. For each $n, A_{0} \subseteq$ $\omega_{n} \subseteq \Lambda\left(f_{n}\right)$ with $A_{0} \subseteq\left[\frac{1}{2}, 1\right]$, yet $\Lambda(f) \cap\left[\frac{1}{2}, 1\right]=\emptyset$.

Example 3[3]. Let $f(x)=x$ on $I$, and for $\epsilon>0$, choose $\frac{1}{n}<\epsilon$. An appropriate polygonal function $f_{n}$ that possesses the orbit $0 \rightarrow \frac{1}{n} \rightarrow \frac{2}{n} \rightarrow$ $\ldots \rightarrow \frac{n-1}{n} \rightarrow 1 \rightarrow \frac{n-\frac{1}{2}}{n} \rightarrow \frac{n-\frac{3}{2}}{n} \rightarrow \ldots \rightarrow \frac{\frac{1}{2}}{n} \rightarrow 0$ has a periodic orbit that spans $I$, and the property that $\left\|f-f_{n}\right\| \leq \frac{1}{n}$. Since $\Omega(f)=\{\{x\}: x \in I\}$, it follows that $\mathcal{H}^{*}\left(\Omega\left(f_{n}\right), \Omega(f)\right)=\frac{1}{2}$ for all $n$. By choosing a subsequence if necessary, one may assume that $\lim _{n \rightarrow \infty} \Omega\left(f_{n}\right)$ exists, since $\left\{\mathcal{K}^{*}, \mathcal{H}^{*}\right\}$ is compact. Then $\lim _{n \rightarrow \infty} f_{n}=f$, and $\mathcal{H}^{*}\left(\lim _{n \rightarrow \infty} \Omega\left(f_{n}\right), \Omega(f)\right)=\frac{1}{2}$. Thus, $\Omega$ is discontinuous at the identity function, a function with zero topological entropy. Unlike Example 2, however, in this example we did not lose any $\omega$ limit points in going from $\Lambda\left(f_{n}\right)$ to $\Lambda(f)$, as $\Lambda(f)=[0,1]$, but we did lose all of our non-trivial $\omega$-limit sets in the limit. 
We should note that both in Example 2 and Example 3, we can take the sequence $\left\{f_{n}\right\}$ to be equicontinuous as well as bounded, so that $\left\{f_{n}\right\}$ has a compact closure in $C(I, I)$. We conclude, then, that $f_{n} \rightarrow f, \omega_{n} \in \Omega\left(f_{n}\right)$ and $\omega_{n} \rightarrow \omega$ do not imply that $\omega$ is in $\Omega(f)$ even for compact sequences $\left\{f_{n}\right\}$.

We begin our next section with a closer examination of the relationship between the limit set $\omega$ and the limit function $f$. We will then revisit the continuity structure of $\Omega$ with our attention restricted to functions of zero topological entropy.

\section{Finite $\omega$-limit sets and zero topological entropy}

Suppose $f_{n} \rightarrow f$ in $C(I, I), \omega_{n} \rightarrow \omega$ in $\mathcal{K}$ and $\omega_{n}$ is an $\omega$-limit set of $f_{n}$ for each $n$. From our previous section's examples we know that $\omega$ need not be an $\omega$-limit set of $f$, although in some cases it might be. The main result of this section shows us that if we have the additional hypothesis that $\omega$ is finite, then $\omega$ will always be an $\omega$-limit set of $f$. Also, if we restrict ourselves to the closed class of functions $\mathcal{E}=\{f \in C(I, I): \mathbf{h}(f)=0\}$, and $\omega$ is infinite, then the necessarily unique maximal perfect subset of $\omega$ is an $\omega$-limit set of $f$. We begin with a couple of lemmas. These show that, regardless of the cardinality of $\omega$ or the topological entropy of the members of $\left\{f_{n}\right\}, \omega$ always possesses certain properties reminiscent of an $\omega$-limit set.

Lemma 4.1. Suppose $f_{n} \rightarrow f, \omega_{n} \rightarrow \omega$ and $\omega_{n} \in \Omega\left(f_{n}\right)$ for each $n$. Then $f(\omega)=\omega$.

Proof. $f(\omega) \subseteq \omega$ : Let $y \in \omega$, and take $\left\{y_{n}\right\}$ so that $y_{n} \in \omega_{n}$ for each $n$, and $y_{n} \rightarrow y$. Then $f_{n}\left(y_{n}\right) \rightarrow f(y)$, and since $f_{n}\left(y_{n}\right) \in \omega_{n}$, it follows that $f(y) \in \omega$.

$\omega \subseteq f(\omega)$ : Let $y \in \omega$, and take $\left\{y_{n}\right\}$ so that $y_{n} \in \omega_{n}$ for each $n$, and $y_{n} \rightarrow y$. Suppose $x_{n} \in f_{n}^{-1}\left(y_{n}\right) \cap \omega_{n}$, with $\left\{x_{n_{k}}\right\} \subseteq\left\{x_{n}\right\}$ a convergent subsequence; say $x_{n_{k}} \rightarrow x$. Then $x \in \omega$, and $f(x)=y$, as $|f(x)-y| \leq\left|f(x)-f\left(x_{n_{k}}\right)\right|+$ $\left|f\left(x_{n_{k}}\right)-f_{n_{k}}\left(x_{n_{k}}\right)\right|+\left|f_{n_{k}}\left(x_{n_{k}}\right)-y\right|$.

Our next lemma shows that not only is $\omega$ strongly invariant with respect to $f$, but also that $f$ transports portions of $\omega$ outside of themselves in a way similar to what $\omega$-limit sets experience.

Lemma 4.2. Suppose $f_{n} \rightarrow f, \omega_{n} \rightarrow \omega$ and $\omega_{n} \in \Omega\left(f_{n}\right)$ for each $n$. If $F$ is any nonempty proper closed subset of $\omega$, then $F \cap \overline{f(\omega \backslash F)} \neq \emptyset$.

Proof. Suppose, to the contrary, that $F$ and $\overline{f(\omega \backslash F)}$ are disjoint. Then there exist open sets $G_{1}, G_{2}$ such that $\omega \backslash F \subseteq G_{1}, F \subseteq G_{2}$ and $\overline{G_{2}}$ is disjoint from $\overline{f\left(G_{1}\right)}$. Say $\sigma=\min \left\{|x-y|: x \in \overline{G_{2}}, y \in \overline{f\left(G_{1}\right)}\right\}$. Since $\omega_{n} \rightarrow \omega$, there exists $M$ a natural number such that $\omega_{n} \subseteq G_{1} \cup G_{2}$ and $\omega_{n} \cap G_{1} \neq \emptyset, \omega_{n} \cap G_{2} \neq \emptyset$ 
for all $n \geq M$. Also, since $f_{n} \rightarrow f$, there is a natural number $N$ so that $\left|f_{m}(x)-f(x)\right|<\frac{\sigma}{2}$ for all $m \geq N$ and $x \in I$. Let us take $n$, then, so that $n>\max \{M, N\}$, and set $F_{n}=\omega_{n} \cap \overline{G_{2}}$. Then $F_{n}$ is a closed, nonempty, proper subset of $\omega_{n}$, and $\overline{G_{2}}$ is disjoint from $\overline{f_{n}\left(G_{1}\right)}$. Let $x_{n} \in I$ so that $\omega_{n}=\omega\left(x_{n}, f_{n}\right)$. For all large $k, f_{n}^{k}\left(x_{n}\right)$ belongs to either $G_{1}$ or $G_{2}$, and it belongs to each of them infinitely often. Thus there is an infinite sequence $k_{1}<k_{2}<k_{3}<\ldots$ so that $f_{n}^{k_{i}}\left(x_{n}\right) \in G_{1}$, and $f_{n}^{k_{i}+1}\left(x_{n}\right) \in G_{2}$. If $y$ is a limit point of the sequence $f_{n}^{k_{i}}\left(x_{n}\right)$, then $y \in \overline{G_{1}}$, and $f(y) \in \overline{G_{2}}$, which is a contradiction.

We are now in a position to show that $\omega$ is an $\omega$-limit set of $f$ whenever $\omega$ is finite.

Theorem 4.1. Suppose $f_{n} \rightarrow f, \omega_{n} \rightarrow \omega$ and $\omega_{n} \in \Omega\left(f_{n}\right)$ for each $n$. If $\omega$ is a finite set, then $\omega \in \Omega(f)$.

Proof. Say $\omega=\left\{z_{1}, z_{2}, \ldots, z_{k}\right\}$. Since $f(\omega)=\omega$, it suffices to show that $f^{j}\left(z_{i}\right) \neq z_{i}$ whenever $1 \leq j<k$ for all $i=1,2, \ldots, k$. Suppose, to the contrary, that there exists $i \in\{1,2, \ldots, k\}$ and $1 \leq j<k$ for which $f^{j}\left(z_{i}\right)=z_{i}$. Let us assume that $f^{l}\left(z_{i}\right) \neq z_{i}$ for all $l<j$. Set $\sigma=\min \left\{\left|z_{m}-z_{p}\right|: m \neq p\right\}$. Since $f_{n} \rightarrow f$, there exists $N_{1} \in \mathbb{N}$ so that $n \geq N_{1}$ implies $\left\|f_{n}-f\right\|<\frac{\sigma}{8}$. Let $N_{2} \in \mathbb{N}$ so that $N_{2}>N_{1}$, and $n \geq N_{2}$ implies $\mathcal{H}\left(\omega, \omega_{n}\right)<\delta<\frac{\sigma}{8}$, where $\delta$ is chosen so that $|a-b|<\delta$ insures that $|f(a)-f(b)|<\frac{\sigma}{8}$. If $n>N_{2}$, and we take $x \in \omega_{n}$ so that $\left|x-z_{l}\right|<\delta$, then $\left|f_{n}(x)-f\left(z_{l}\right)\right| \leq\left|f_{n}(x)-f(x)\right|+$ $\left|f(x)-f\left(z_{l}\right)\right| \leq \frac{\sigma}{8}+\frac{\sigma}{8}=\frac{\sigma}{4}$. It now follows that if $\omega_{n}^{*}=\omega_{n} \cap B_{\delta}\left(\cup_{l=0}^{j} f^{l}\left(z_{i}\right)\right)$, then $f_{n}\left(\omega_{n}^{*}\right) \subseteq \omega_{n}^{*}$. If we let $F=\omega_{n} \backslash \omega_{n}^{*}$, then $F \cap \overline{f_{n}\left(\omega_{n} \backslash F\right)}=\emptyset$. But this contradicts our hypothesis that $\omega_{n}$ is an $\omega$-limit set for $f_{n}$.

In our previous result we were able to show that $\omega$ is an $\omega$-limit set of $f$ provided we place a restriction on the structure of $\omega$. We are able to get a similar result by placing a restriction on the sequence $\left\{f_{n}\right\}$ rather than on the limit set $\omega$. We restrict our attention to $\mathcal{E}=\{f \in C(I, I): \mathbf{h}(f)=0\}$, and after showing that $\mathcal{E}$ is closed in $\{C(I, I),\|\|$.$\} , we go on to prove the following$ theorem.

Theorem 4.2. Suppose $\left\{f_{n}\right\} \subseteq \mathcal{E}, f_{n} \rightarrow f, \omega_{n} \rightarrow \omega$ and $\omega_{n} \in \Omega\left(f_{n}\right)$ for each $n$. If $\omega$ is infinite and $C$ is the set of isolated points of $\omega$, then $\omega \backslash C \in \Omega(f)$.

We begin our proof of this theorem with a verification that our set $\mathcal{E}$ is indeed closed.

Lemma 4.3. The set $\mathcal{E}=\{f \in C(I, I): \mathbf{h}(f)=0\}$ is closed in $\{C(I, I),\|\|$.$\} .$

Proof. From [1], we know that the function $\mathbf{h}: C(I, I) \rightarrow \mathbf{R}^{+} \cup\{+\infty\}$, given by $f \rightarrow \mathbf{h}(f)$, where $\mathbf{h}(f)$ is the topological entropy of $f$, is lower semicontinuous. Thus, if $\mathbf{h}(f)>\alpha>0$, then $\mathbf{h}(g)>\alpha$ for all $g$ sufficiently close to $f$. 
In particular, then, the set $G=\{f \in C(I, I): \mathbf{h}(f)>0\}$ is open, and our conclusion follows.

Our next lemma, though rather technical, plays an important part in the proof of Theorem 4.2.

Lemma 4.4. Suppose $\left\{f_{n}\right\} \subseteq \mathcal{E}, f_{n} \rightarrow f, \omega_{n} \rightarrow \omega$ and $\omega_{n} \in \Omega\left(f_{n}\right)$ for every $n$. If $\omega$ is infinite and $x \in \omega$, then $x$ is not a periodic point of $f$.

Proof. Since $\mathbf{h}(f)=0$, if $x$ is periodic, the period of $x$ is $2^{m}$ for some $m$ in $\mathbb{N} \cup\{0\}$. Thus, $x$ is a fixed point of the function $g=f^{2^{m}}$, and since $f_{n} \rightarrow f$, it follows that $g_{n}=f_{n}^{2^{m}} \rightarrow g$. Suppose $x_{n} \rightarrow x$ and $x_{n}$ is periodic of period $2^{n}$. Let $n \geq m$. Then $x_{n}$ is periodic of period $2^{n-m}$ for $g_{n}$, and $x \in \widetilde{\omega}$, the limit of any convergent subsequence $\left\{\omega\left(x_{n_{k}}, g_{n_{k}}\right)\right\} \subseteq\left\{\omega\left(x_{n}, g_{n}\right)\right\}$. It suffices, then, to show that $x$ is not a fixed point of $f$, for if $x$ has a period of $2^{m}, m>0$, we replace the sequence $\left\{f_{n}\right\}$ with $\left\{g_{n}\right\}=\left\{f_{n}^{2^{m}}\right\}$, and the sequence $\left\{\omega_{n}\right\}$ with a convergent subsequence of $\left\{\omega\left(x_{n}, g_{n}\right)\right\}$. We proceed in two cases.

Case 1: Suppose $\omega_{n}$ is finite for each $n$. By renumbering and taking a subsequence if necessary, we may assume that $\left|\omega_{n}\right|=2^{n}$ for each $n$. We prove our first case by again considering two possibilities.

First, let us suppose that $x=\min \omega$ or $x=\max \omega$ is a fixed point. We will prove our assertion in the case that $x=\min \omega$. Let $x_{n}=\min \omega_{n}$, and since $\omega_{n} \rightarrow \omega$, it follows that $x_{n} \rightarrow x$. Since $\left\{f_{n}\right\} \cup f$ is a closed and bounded set in $C(I, I)$, we know that it is equicontinuous. Let $\epsilon>0$, and take $\epsilon>\delta>0$ so that $|a-b|<\delta$ implies $|g(a)-g(b)|<\epsilon$ for any $g \in\left\{f_{n}\right\} \cup f$. Let $N \in \mathbb{N}$ so that $n>N$ implies $\left|x_{n}-x\right|<\delta$, and $\left|f_{n}\left(x_{n}\right)-x_{n}\right|<\delta$, too. Since $x_{n}=\min \omega_{n}, f_{n}\left(x_{n}\right) \geq x_{n}$, and since $f^{-1}\left(\max \omega_{n}\right) \leq f_{n}\left(x_{n}\right)$, it follows that $\overline{\operatorname{conv}} \omega_{n} \subset B_{2 \epsilon}(x)$. Thus, $\left|\overline{\operatorname{conv}} \omega_{n}\right| \rightarrow 0$ as $n \rightarrow \infty$. This implies $x=\omega$, a contradiction.

Now, let us suppose that $x \in(\min \omega, \max \omega)$, and again we will take $\epsilon>$ $\delta>0$ so that $|a-b|<\delta$ implies $|g(a)-g(b)|<\epsilon$ for each $g \in\left\{f_{n}\right\} \cup$ $f$. Suppose that $x_{n} \in \omega_{n}$ and $x_{n} \rightarrow x$. Choose $N \in \mathbb{N}$ so that $n>N$ implies $\left|x-x_{n}\right|<\frac{\delta}{2}$, and $\left|f_{n}^{i}\left(x_{n}\right)-x_{n}\right|<\frac{\delta}{2}$ for $i=0,1,2,3$. Set $a=$ $\min \left\{f_{n}^{i}\left(x_{n}\right)\right\}_{i=0}^{3}$ and $b=\max \left\{f_{n}^{i}\left(x_{n}\right)\right\}_{i=0}^{3}$. Then $[a, b]$ contains at least one of $f_{n}^{-1}\left(\max \omega_{n}\right), f_{n}^{-1}\left(\min \omega_{n}\right)$ so that either $\left|\max \omega_{n}-x\right|<2 \varepsilon$ or $\left|\min \omega_{n}-x\right|<$ $2 \epsilon$. Thus we have either that $\left|\max \omega_{n}-x\right| \rightarrow 0$ or $\left|\min \omega_{n}-x\right| \rightarrow 0$ as $n \rightarrow \infty$. In either case we have a contradiction to our supposition that $x \in\left(\min \omega_{n}, \max \omega_{n}\right)$.

Case 2: Suppose $\omega_{n}$ is uncountable for all $n>N$, for some $N \in \mathbb{N}$.

Let $J_{i}^{k}, i=1,2$, be the rank 1 periodic portions of $\omega_{k}$ with respect to $f_{k}$. Since $\omega_{k} \rightarrow \omega$ and $f_{k} \rightarrow f$, it follows that $\left\{J_{1}^{k}\right\}$ and $\left\{J_{2}^{k}\right\}$ each converges to a portion $J_{1}$ and $J_{2}$, respectively, of $\omega$ that $f$ exchanges. Since $\omega=J_{1} \cup J_{2}$, 
if $x \in J_{1}$, then $f(x) \in J_{2}$, so $f(x)=x$ implies $J_{1} \cap J_{2} \neq \emptyset$. By considering the four rank 2 periodic portions of $\omega$, however, one sees that $J_{1} \cap J_{2} \neq \emptyset$ is impossible.

Using the notation of Theorem 4.2, Theorem 5.1[7] tells us that it suffices to show that $\omega \backslash C$ is a subset of the limit points of $f$, and $\omega \backslash C$ is both simple and strongly invariant with respect to $f$ in order to prove our result.

Lemma 4.5. Suppose $\left\{f_{n}\right\} \subseteq \mathcal{E}, f_{n} \rightarrow f, \omega_{n} \rightarrow \omega$ and $\omega_{n} \in \Omega\left(f_{n}\right)$ for every $n$. If $\omega$ is infinite and $C$ is the set of isolated points of $\omega$, then

- $\omega \backslash C$ is a simple set with respect to $f$,

- $\omega \backslash C \subseteq \Lambda(f)$, and

- $f(\omega \backslash C)=\omega \backslash C$.

Proof. We prove our result in several steps.

1. We first show that $\omega$ is a simple set with respect to $f$. Suppose $\omega_{m}$ is a periodic orbit of $f_{m}$ of order $2^{m}$, or that $\omega_{m}$ is uncountable. In either case, if $m>2$, there exist two disjoint compact portions $J_{1}^{m}, J_{2}^{m}$ of $\omega_{m}$ that $f_{m}$ interchanges. Since $\omega_{m} \rightarrow \omega$ and $f_{m} \rightarrow f$, it follows that $J_{1}^{m} \rightarrow J_{1}, J_{2}^{m} \rightarrow J_{2}$, and $J_{1}$ and $J_{2}$ are interchanged by $f$. Moreover, since $J_{1}^{m} \cup J_{2}^{m} \supseteq \omega_{m}$, one sees that $J_{1} \cup J_{2} \supseteq \omega$, and they are disjoint since no element of $\omega$ can be periodic. In a similar fashion one can show that, since $J_{1}^{m}$ and $J_{2}^{m}$ both have a disjoint decomposition into two compact subportions that $f_{m}^{2}$ interchanges, the same is true of $J_{1}, J_{2}$ and $f$.

2. We now show that $\omega \backslash C$ is contained in $\Lambda(f)$. Let $x \in \omega \backslash C$. Since $\omega$ is a simple set, there exists a nested sequence of compact periodic portions $\left\{J_{k}\right\}_{k=1}^{\infty}$ such that the period of $J_{k}$ is $2^{k}$ for each $k$, and $x$ is contained in each. Let $J=\cap_{k=1}^{\infty} J_{k}$. We begin by showing that $x$ is not contained in $\operatorname{int}(\overline{\operatorname{conv}}(J))$, the interior of the convex closure of $J$. Suppose, to the contrary, that there exists $\delta>0$ such that $B_{\delta}(x) \cap \operatorname{int}(\overline{\operatorname{conv}}(J))=B_{\delta}(x)$. Then for sufficiently large $k, B_{\delta}(x)$ is contained in the interior of a component of the simple system for $\omega_{k}$. From Proposition 3.1[5], it follows that $B_{\delta}(x) \cap \omega_{k}=\emptyset$, which contradicts $x$ being an element of $\omega=\lim _{k \rightarrow \infty} \omega_{k}$. We continue our proof by considering two cases.

First, suppose $x=J$. Since each $J_{k}$ is periodic with period $2^{k}, \overline{\operatorname{conv}} J_{k}$ contains a point of period $2^{k}$. Since $J_{k} \rightarrow x$, and $\Lambda(f)$ is closed, our conclusion follows.

Now, suppose $x=\min J$ or $x=\max J$. Let us assume that $x=\max J$. Then there exists $\left\{y_{n}\right\} \subseteq \omega$ such that $x<y_{n}$ for all $n$, and $y_{n} \rightarrow x$. Since there is a periodic point between any two of the compact periodic portions of rank $k$ for each $k$, our conclusion follows. 
3. We show that $\omega \backslash C$ is strongly invariant with respect to $f$. This, in conjunction with our first step, also establishes that $\omega \backslash C$ is a simple set with respect to $f$. Let $x \in \omega \backslash C$ with $\left\{y_{n}\right\} \subseteq \omega \backslash C$ such that $y_{n} \rightarrow x$. Since $f$ is continuous and $x$ is not an element of $\operatorname{int}(\overline{\operatorname{conv}}(J))$, we have that $f\left(y_{n}\right) \rightarrow f(x)$. Thus, $f(x)$ is not an isolated point of $\omega$, so that $f(x) \in \omega \backslash C$, and more generally, $f(\omega \backslash C) \subseteq \omega \backslash C$. Now, let $y \in \omega \backslash C$, and suppose $\left\{y_{n}\right\}$ is a subset of $\omega \backslash C$ such that $y_{n} \rightarrow y$. Take $x_{n} \in\left\{f^{-1}\left(y_{n}\right)\right\} \cap \omega$, and let $\left\{x_{n_{k}}\right\}$ be a convergent subsequence of $\left\{x_{n}\right\}$; say $x_{n_{k}} \rightarrow x$. Then $x \in \omega$ since $\omega$ is closed, and as $x$ is not isolated, $x$ is not contained in $C$. Since $f$ is continuous, $x_{n_{k}} \rightarrow x$ and $f\left(x_{n_{k}}\right) \rightarrow y$ imply that $f(x)=y$, and it follows that $f(\omega \backslash C) \supseteq \omega \backslash C$.

We would like to be able to extend the conclusion of Theorem 4.2 to the entire set $\omega$, thereby establishing the semicontinuity of the map $\Omega \mid \mathcal{E}$. Using a construction found in [5], however, we are able to develop a sequence of functions $\left\{f_{n}\right\} \subseteq C(I, I)$ so that $\mathbf{h}\left(f_{n}\right)=0$ for each $n$, there is a set $\omega$ contained in $\Omega\left(f_{n}\right)$ for each $n$, and $g=\lim _{n \rightarrow \infty} f_{n}$ exists; nevertheless, $\omega$ is not an element of $\Omega(g)$. We note that $\omega$ must necessarily be of the form $Q \cup C$, where $Q$ is a Cantor set, and $C$ is a countable set of points isolated in $\omega$. This construction of Bruckner and Ceder can be thought of as a reversal of Smital's Theorem in that their function $f$ is developed for a particular sequence of compact periodic intervals $\left\{J_{k}\right\}$.

Prior to developing Bruckner and Ceder's example, we recall a device from [8] that allows us to code the sets $f^{i}\left(J_{k}\right)$ found in Smital's Theorem with finite tuples of zeros and ones. Let $\mathbb{N}$ denote the natural numbers, and take $\mathcal{N}$ to be the set of sequences composed of zeros and ones. If $\mathbf{n} \in \mathcal{N}$ and $\mathbf{n}=\left\{n_{i}\right\}_{i=1}^{\infty}$, we let $\mathbf{n} \mid k=\left(n_{1}, n_{2}, \ldots, n_{k}\right)$. Set $\mathbf{0}=\{0,0,0, \ldots\}$ and $\mathbf{1}=\{1,1,1, \ldots$.$\} .$ Now, define a function $\mathcal{A}: \mathcal{N} \rightarrow \mathcal{N}$ given by $\mathcal{A}(\mathbf{n})=\mathbf{n}+10$, where addition is modulus two from left to right. For each $k \in \mathbb{N}$ and $i \in \mathbb{N}$ put $F_{\mathbf{1} \mid k}=J_{k}$ and $F_{\mathcal{A}^{i}(\mathbf{1} \mid k)}=f^{i}\left(J_{k}\right)$. Thus, for every $\mathbf{m}$ and $\mathbf{n}$ in $\mathcal{N}$ and $k \in \mathbb{N}$ there is a $j \in \mathbb{N}$ such that $\mathcal{A}^{j}(\mathbf{m} \mid k)=\mathbf{n} \mid k$; the above relations define $F_{\mathbf{n} \mid k}$ for all $\mathbf{n} \in \mathcal{N}$ and $k \in \mathbb{N}$. In the construction that follows, we will take $F_{\mathbf{n} \mid k, 1}$ to lie to the left of $F_{\mathbf{n} \mid k, 0}$ for all $\mathbf{n} \in \mathcal{N}$ and $k \in \mathbb{N}$.

Example 4. Let $Q$ be any Cantor set in $(0,1)$, and let $C$ be a set that consists of exactly one point from each interval contiguous to $Q$ together with the point $\frac{1}{2} \inf Q$. We let $\mathcal{M}$ consist of all $\mathbf{n} \in \mathcal{N}$ that have a tail of ones. We define by induction a system of closed intervals $\left\{F_{\mathbf{n} \mid k}: \mathbf{n} \in \mathcal{N}, k \in \mathbb{N}\right\}$ so that for each $\mathbf{n}$ and $k, F_{\mathbf{n} \mid k, 1}$ and $F_{\mathbf{n} \mid k, 0}$ are disjoint subintervals contained in the interior of $F_{\mathbf{n} \mid k}$ for which the nondegenerate components of $K=\cup_{\mathbf{n} \in \mathcal{N}} \cap_{k=1}^{\infty}$ $F_{\mathbf{n} \mid k}$ coincide with all $F_{\mathbf{n}}=\cap_{k=1}^{\infty} F_{\mathbf{n} \mid k}$ with $\mathbf{n} \in \mathcal{M}$. These in turn coincide with all $[c, q]$ where $c \in C$ and $q$ is the nearest point to the right of $c$ in $Q$. We 
also choose $F_{1}$ and $F_{0}$ so that $0=\inf F_{1}$ and $1=\sup F_{0}$.

For each $\mathbf{n} \in \mathcal{N}$ and $k \in \mathbb{N}$ let $F_{\mathbf{n} \mid k}=\left[a_{\mathbf{n} \mid k}, b_{\mathbf{n} \mid k}\right]$. If $\mathbf{n} \in \mathcal{M}$, then $\cap_{k=1}^{\infty}\left[a_{\mathbf{n} \mid k}, b_{\mathbf{n} \mid k}\right]=\left[a_{\mathbf{n}}, b_{\mathbf{n}}\right]$ where $a_{\mathbf{n}}$ and $b_{\mathbf{n}}$ are the endpoints of $F_{\mathbf{n}}$; the set $C$ consists of all $a_{\mathbf{n}}$ for which $\mathbf{n} \in \mathcal{M}$. Let $S$ consist of all $x$ such that $\{x\}=F_{\mathbf{n}}$ for some $\mathbf{n} \in \mathcal{N}$. Then the closure of $S$ is $Q$, and if $B$ consists of all $b_{\mathbf{n}}$ for which $\mathbf{n} \in \mathcal{M}$, then $Q=S \cup B$. We also note that $F_{\mathcal{A}(\mathbf{n})}$ is a singleton whenever $F_{\mathbf{n}}$ is a singleton.

Let $L=Q \cup C \cup\left\{a_{\mathbf{n} \mid k}: \mathbf{n} \in \mathcal{N}, k \in \mathbb{N}\right\} \cup\left\{b_{\mathbf{n} \mid k}: \mathbf{n} \in \mathcal{N}, k \in \mathbb{N}\right\}$. We first define our function $f$ on $L$. If $x \in S$, define $f(x)$ so that $\{f(x)\}=F_{\mathcal{A}(\mathbf{n})}$ when $\{x\}=F_{\mathbf{n}}$, and on $C \cup B$ define $f$ so that $f\left(a_{\mathbf{n}}\right)=a_{\mathcal{A}(\mathbf{n})}$ and $f\left(b_{\mathbf{n}}\right)=b_{\mathcal{A}(\mathbf{n})}$. On the remaining points of $L$ we set $f\left(a_{\mathbf{n} \mid k}\right)=a_{\mathcal{A}(\mathbf{n}) \mid k}$ and $f\left(b_{\mathbf{n} \mid k}\right)=b_{\mathcal{A}(\mathbf{n}) \mid k}$ whenever $\mathbf{n}|k \neq \mathbf{1}| k$, and take $f\left(a_{\mathbf{1} \mid k}\right)=a_{\mathbf{0} \mid k+1}$ and $f\left(b_{\mathbf{1} \mid k}\right)=b_{\mathbf{0} \mid k+1}$.

Bruckner and Ceder show that the function $f: L \rightarrow L$ is continuous, and then extend $f$ linearly on the intervals contiguous to $L$ obtaining a function also denoted by $f$ that is continuous on all of $[0,1]$. They go on to show that $f$ has exactly one $2^{k}$ cycle for each $k \in \mathbb{N} \cup\{0\}$, but no other periodic points, so that $\mathbf{h}(f)=0$. Since the orbit of the point $a_{0}$ is $\left\{a_{\mathbf{n} \mid k}: \mathbf{n} \in \mathcal{N}, k \in \mathbb{N}\right\}$, it follows that $Q \cup C=\omega\left(a_{0}, f\right) \in \Omega(f)$.

Suppose $c \neq a_{\mathbf{1}}$ is an isolated point of $Q \cup C$, and $c$ is contained in $(b, a)$ an interval contiguous to $Q$. We call $(x, c)$, for $b<x<c$, an exterior neighborhood of $c$. If $c=a_{\mathbf{1}}$, we take $(x, c)$ to be an exterior neighborhood of $c$ for any $x \in[0, c)$. The idea behind the development of our functions $f_{n}$ is to modify Bruckner and Ceder's function $f$ so that the trajectory of $a_{0}$ intersects the exterior neighborhoods of the set $C$ less and less frequently. In the limit $g=\lim _{n \rightarrow \infty} f_{n}$, we will see that the trajectory of any point $x$ in $[0,1]$ can visit an exterior neighborhood of $a_{1}$ at most one time.

We now define our sequence of functions $\left\{f_{n}\right\}$. Let $f_{1}$ be Bruckner and Ceder's function $f$, and define $f_{2}$ so that

- $f_{2}(x)=f(x)$ for $x \geq a_{1}$,

- $f_{2}\left(a_{1 \mid k}\right)=a_{\mathbf{0} \mid k+2}$ for all $k$ in $\mathbb{N}$,

and extend $f_{2}$ linearly on $\left[0, a_{1}\right)$. This extension is possible since $a_{0}=$ $\lim _{k \rightarrow \infty} a_{\mathbf{0} \mid k+2}=\lim _{k \rightarrow \infty} f_{2}\left(a_{\mathbf{1} \mid k}\right)=f_{2}\left(a_{\mathbf{1}}\right)$. In general we take $f_{l}$ so that $f_{l}(x)=f(x)$ for $x \geq a_{\mathbf{1}}$ and $f_{l}\left(a_{\mathbf{1} \mid k}\right)=a_{\mathbf{0} \mid k+l}$ for all $k \in \mathbb{N}$, and then extend $f_{l}$ linearly on $\left[0, a_{\mathbf{1}}\right)$.

It follows that each of our functions $f_{n}$ satisfies Bruckner and Ceder's Theorem 4.3 - in fact, their proof carries over except for the obvious changes in notation - so that $f_{n}$ has zero topological entropy, and $Q \cup C=\omega\left(a_{0}, f_{n}\right) \in$ $\Omega\left(f_{n}\right)$, since $\left\{a_{\mathbf{n} \mid 1+n k}: \mathbf{n} \in \mathcal{N}, k \in\{0\} \cup \mathbb{N}\right\}$ is the orbit of the point $a_{0}$. 
If we let $g=\lim _{n \rightarrow \infty} f_{n}$, however, one sees that $g(x)=a_{\mathbf{0}}$ for all $x$ contained in $\left[0, a_{1}\right]$, so that the orbit of any point contained in the unit interval visits an exterior neighborhood of $a_{1}$ at most once. It follows that $a_{1}$ cannot be contained in any $\omega$-limit set of $g$; in particular, $Q \cup C$ cannot be an $\omega$-limit set of $g$. As Theorem 4.2 indicates, however, $Q$ - the maximal perfect subset of $\omega$ - is an $\omega$-limit set of $g$. In fact, $Q=\omega\left(a_{0}, g\right)$.

\section{Continuity of $\Omega$ when $\Omega(f)$ is finite}

In this section we return to a study of the map $\Omega:\{C(I, I),\|\|.\} \rightarrow\left\{\mathcal{K}^{*}, \mathcal{H}^{*}\right\}$. Our main result shows that if $f$ has only a finite number of stable $\omega$-limit sets, then $f$ is a point of continuity of the map $\Omega$. Again, we begin with a series of lemmas.

Lemma 5.1. Suppose $f$ in $C(I, I)$ has only a finite number of $\omega$-limit sets; say that $|\Omega(f)|=m$. Then $\Omega(f)$ is a finite number of periodic $\omega$-limit sets, each with cardinality a power of two, and $\mathbf{h}(f)=0$.

Proof. Since $\Omega(f)$ is finite, it follows from Sarkovskii's Theorem that $\mathbf{h}(f)=$ 0 . If $f$ has an uncountable $\omega$-limit set, then $f$ has a $2^{n}$ orbit for each $n \in \mathbb{N}$, which contradicts $|\Omega(f)|=m$. Thus, if $\omega$ is an $\omega$-limit set of $f$, then $|\omega|=2^{n}$ for some $n \leq m-1$.

Lemma 5.2. Suppose $f$ is in $C(I, I),|\Omega(f)|=n$ and each of the $\omega$-limit sets of $f$ is stable. If for any $\epsilon>0$ there exists $\delta>0$ so that $\|f-g\|<\delta$ implies $\Lambda(g) \subseteq B_{\epsilon}(\Lambda(f))$, then the map $\Omega:\{C(I, I),\|\|.\} \rightarrow\left\{\mathcal{K}^{*}, \mathcal{H}^{*}\right\}$ is continuous at $f$.

Proof. We show that for each $\epsilon>0$ there is a $\delta>0$ so that $\mathcal{H}^{*}(\Omega(g), \Omega(f))$ $<\epsilon$ whenever $\|f-g\|<\delta$. Let $\epsilon>0$, take $\left\{x_{1}, x_{2}, \ldots, x_{k}\right\}=\Lambda(f)$ such that $i<j$ implies $x_{i}<x_{j}$, and set $\gamma=\min \left\{x_{1}, x_{i+1}-x_{i}, 1-x_{k}: i=1,2, \ldots, k-1\right\}$. We may assume $\epsilon<\frac{\gamma}{3}$. Since $f$ is uniformly continuous, there exists $\delta_{1}, 0<$ $\delta_{1}<\frac{\epsilon}{3}$, so that $|x-y|<\delta_{1}$ implies $\left|f^{i}(x)-f^{i}(y)\right|<\frac{\epsilon}{3}$ for $i=1,2, \ldots, 2^{n-1}$. Let $\sigma_{1}=\min \left\{\left|f^{2^{n-1}}(x)-x\right|: x \in I \backslash B_{\delta_{1}}(\Lambda(f))\right\}$, and set $\sigma=\min \left\{\delta_{1}, \frac{\sigma_{1}}{2}\right\}$. Let $\delta_{2}>0$ so that $\|f-g\|<\delta_{2}$ implies $\left\|f^{i}-g^{i}\right\|<\sigma$ for $i=1,2, \ldots, 2^{n-1}$. By hypothesis, there exists $\delta, 0<\delta<\delta_{2}$ such that $\|f-g\|<\delta$ implies $\Lambda(g) \subseteq B_{\delta_{2}}(\Lambda(f)) \subseteq B_{\epsilon}(\Lambda(f))$. Let $\omega_{i}=\omega\left(x_{i}, f\right)$, and take $x_{i}^{*}$ in $B_{\delta_{2}}\left(x_{i}\right)$ so that $g^{2^{n-1}}\left(x_{i}^{*}\right)=x_{i}^{*}$. Then $\left|g^{m}\left(x_{i}^{*}\right)-f^{m}\left(x_{i}\right)\right| \leq\left|g^{m}\left(x_{i}^{*}\right)-f^{m}\left(x_{i}^{*}\right)\right|+$ $\left|f^{m}\left(x_{i}^{*}\right)-f^{m}\left(x_{i}\right)\right|<\epsilon$ for $m=1,2, \ldots, 2^{n-1}$, so $\mathcal{H}\left(\omega\left(x_{i}^{*}, g\right), \omega_{i}\right)<\epsilon$. Moreover, since $\left|g(x)-f\left(x_{i}\right)\right|<\frac{\gamma}{3}$ for all $x$ in $B_{\delta_{2}}\left(x_{i}\right)$, and $\omega_{g} \in \Omega(g)$ implies $g\left(\omega_{g}\right)=\omega_{g}$, it follows that $\mathcal{H}\left(\omega_{g}, \omega_{i}\right)<\epsilon$ whenever $\omega_{g} \cap B_{\delta_{2}}\left(x_{i}\right) \neq \emptyset$. 
Lemma 5.3. Suppose $f$ is in $C(I, I),|\Omega(f)|=n$ and each of the $\omega$-limit sets of $f$ is stable. Then for any $\epsilon>0$ there exists $\delta>0$ so that $\|f-g\|<\delta$ implies $P(g) \subseteq B_{\epsilon}(\Lambda(f))$, where $P(g)$ is the set of periodic points of $g$.

Proof. We begin with some notation that will be helpful in the course of our proof. Suppose that $\omega$ is a periodic $\omega$-limit set for $g \in C(I, I)$, and set $x_{M}=\max \omega, x_{m}=\min \omega$, and take $x_{M-i}$ in $\omega$ so that $g^{i}\left(x_{M-i}\right)=x_{M}$. We define $x_{m-i}$ analogously. Let $[a, b] \rightarrow[c, d]$ indicate that $g([a, b]) \supseteq[c, d]$; we write $\langle c, d\rangle$ to represent the closed interval with endpoints $c$ and $d$, not caring whether $c \geq d$ or $c \leq d$. Finally, let Fix $(g)=\{x \in I: g(x)=x\}$ be the set of fixed points of $g$.

By considering $f^{2^{n-1}}$ rather than $f$ if necessary, we may assume that $\Omega(f)=\left\{\left\{x_{1}\right\},\left\{x_{2}\right\}, \ldots,\left\{x_{m}\right\}\right\}$. Let $\sigma_{1}=\min \left\{|f(x)-x|: x \in I \backslash \cup_{i=1}^{m} B_{\delta}\left(x_{i}\right)\right\}$ and $\sigma_{2}=\min \left\{\left|f^{2}(x)-x\right|: x \in I \backslash \cup_{i=1}^{m} B_{\delta}\left(x_{i}\right)\right\}$, and set $\sigma=\min \left\{\sigma_{1}, \sigma_{2}\right\}$. If $\|f-g\|<\sigma$, then $g$ has no fixed points or points of period two in $I \backslash \cup_{i=1}^{m} B_{\delta}\left(x_{i}\right)$. We proceed in several steps.

1. Suppose $g_{n} \rightarrow f$ and $\omega_{n}=\omega\left(x^{n}, g_{n}\right)$ is periodic. Let us also suppose that $x_{i}$ is a fixed point of $f$, and there exists an $M$ in $\mathbb{N}$ so that for any $\epsilon>0$ there exists $N \in \mathbf{N}$ such that $x_{M-m}^{n} \in\left(x_{i}-\epsilon, x_{i}+\epsilon\right)$ for all $\omega_{n}$, where $n>N$. Since $g_{n} \rightarrow f$ and $x_{M-m}^{n} \rightarrow x_{i}$, it follows that $g_{n}^{m}\left(x_{M-m}^{n}\right)=x_{M}^{n}$ converges to $f^{m}\left(x_{i}\right)=x_{i}$. It follows that $\omega_{n} \rightarrow x_{i}$.

2. Let $x_{i}$ be the fixed point of $f$ contained in $\overline{\operatorname{conv}} \omega$, and suppose $y \in$ $\left[x_{i}+\delta, x_{M}\right]$ such that $g(y) \geq x_{i}^{*} \in F i x(g)$. From our first part we may assume that $x_{m-1}>x_{i}+\delta$, and that $x_{M-1}<x_{i}+\delta$. Then $\left\langle y, x_{m-1}\right\rangle \rightarrow$ $\left[x_{m}, x_{i}^{*}\right] \rightarrow\left\langle y, x_{m-1}\right\rangle$, which implies $\left\langle y, x_{m-1}\right\rangle$ contains a point of period two. But this contradicts $\|f-g\|<\sigma$. We conclude that if $y \in\left[x_{i}+\delta, x_{M}\right]$, then $g(y)<\min \{$ Fix $(g)\}$. Similarly, $y$ in $\left[x_{m}, x_{i}-\delta\right]$ implies $g(y)>\max \{$ Fix $(g)\}$.

3 . We show that $x_{m} \neq x_{M-1}$, and $x_{M} \neq x_{m-1}$. Suppose, to the contrary, that $x_{m}=x_{M-1}$ for $\omega=\omega(x, g)$ where $\|f-g\|<\sigma$. Let $x_{i}$ be the fixed point of $f$ contained in $\overline{c o n v} \omega$, and again we may assume that $x_{m-1}>x_{i}+\delta$. If $x_{i}+\delta \leq y<x_{m-1}$, then $\left[y, x_{m-1}\right] \rightarrow\left[x_{m}, g(y)\right] \rightarrow\left[g^{2}(y), x_{M}\right]$, which implies $g^{2}(y)>y$ since we have no point of period two in $I \backslash \cup_{i=1}^{m} B_{\delta}\left(x_{i}\right)$. Since $g$ is continuous, it follows that $g^{2}(y)>y$ for all $y$ in $\left[x_{i}+\delta, x_{M}\right]$. But $g^{2}\left(x_{M}\right)<x_{M}$, since $|\omega|>2$, which is a contradiction.

4. From our first part, we again assume that $x_{M-1}\left\langle x_{i}-\delta\right.$ and $\left.x_{m-1}\right\rangle$ $x_{i}+\delta$. Let $y \in\left[x_{m}, x_{i}-\delta\right]$. Since $g^{2}$ is continuous, and $g^{2}\left(x_{m}\right)>x_{m}$, it follows that $g^{2}(y)>y$. Similarly, $y \in\left[x_{i}+\delta, x_{M}\right]$ implies $g^{2}(y)<y$. Now, set $x^{*}=\min \left\{x \in \omega: x \in\left[x_{i}+\delta, x_{M}\right]\right\}$. Then $x^{*} \leq x_{m-1}<x_{M}$, and $g^{2}\left(x^{*}\right)<x^{*}$. Let $I_{1}=\left[x^{*}, x_{M}\right]$, so that $x_{m-1} \in I_{1}$, and $I_{2}=g\left(I_{1}\right) \supseteq\left[x_{m}, g\left(x^{*}\right)\right]$. We consider two cases.

Case 1: Suppose $x_{M-1}$ is in $I_{2}$. Then $I_{1} \rightarrow I_{2} \rightarrow I_{1}$, which implies there 
is $y$ in $I_{1}$ such that $g^{2}(y)=y$, a contradiction.

Case 2: Suppose $x_{M-1}$ is not an element of $I_{2}$. Then $g\left(x^{*}\right)<x_{M-1}$. Let $I_{1}^{*}=\left[g\left(x^{*}\right), x_{M-1}\right]$. Then $I_{2}^{*}=\left[g^{2}\left(x^{*}\right), x_{M}\right] \supset \omega \cap\left[x_{i}+\delta, x_{M}\right]$. If $x_{M-2} \in I_{2}^{*}$, then there is a $y$ in $I_{1}^{*}$ such that $g^{2}(y)=y$. Thus, $x_{M-2}$ is contained in $B_{\delta}\left(x_{i}\right)$, which implies $\omega \rightarrow x_{i}$ as $\delta \rightarrow 0$, from part 1 .

We are now in a position to prove the main result of this section. In the course of the proof we will need the following result of Block and Coppel [1].

Lemma 5.4. Let $G$ be an open subinterval which contains no periodic point of $f \in C(I, I)$. Then $G$ contains at most one point of any $\omega$-limit set of $f$.

Theorem 5.1. Suppose $f \in C(I, I),|\Omega(f)|=n$ and each of the $\omega$-limit sets of $f$ is stable. Then the map $\Omega:\{C(I, I),\|\|.\} \rightarrow\left\{\mathcal{K}^{*}, \mathcal{H}^{*}\right\}$ is continuous at $f$.

Proof. Since $|\Omega(f)|=n$, we know from Lemma 5.1 that $\max \left\{\left|\omega_{i}\right|: \omega_{i} \in\right.$ $\Omega(f)\} \leq 2^{n-1}$, so that $f^{2^{n-1}}$ has $\sum_{i=0}^{n-1} 2^{o(i)}$ fixed points in $\Omega\left(f^{2^{n-1}}\right)$, where $2^{o(i)}=\left|\omega_{i}\right|$. If there exists a $\delta>0$ so that $\|f-g\|<\delta$ implies $\Lambda\left(g^{2^{n-1}}\right) \subseteq$ $B_{\epsilon}\left(\Lambda\left(f^{2^{n-1}}\right)\right)$, then $\Lambda(g) \subseteq B_{\epsilon}(\Lambda(f))$ since $\Lambda(h)=\Lambda\left(h^{k}\right)$ for any $h \in C(I, I)$ and $k \in \mathbb{N}$. Moreover, this would allow us to assert our conclusion. We can assume, then, that $\Omega(f)=\left\{\left\{x_{0}\right\},\left\{x_{1}\right\}, \ldots,\left\{x_{n-1}\right\}\right\}$, where $i<j$ implies $x_{i}<$ $x_{j}$, and $x_{i}$ is not a tangential $\omega$-limit set for any $i$. It suffices to show that for any $\epsilon>0$ there exists $\delta>0$ such that $\|f-g\|<\delta$ implies $\mathcal{H}(\Lambda(g), \Lambda(f))<\epsilon$. Let us suppose this is not the case. Say there exists $\left\{g_{n}\right\} \subset C(I, I)$ so that $g_{n} \rightarrow f, \omega_{n} \in \Omega\left(g_{n}\right)$ and $\omega_{n} \rightarrow \omega$ which is not contained in $B_{\epsilon}(\Lambda(f))$. Then $f(\omega)=\omega$, and $\omega$ must be infinite. Let $x \in \omega$. Then $\left\{f^{n}(x)\right\}_{n=0}^{\infty} \rightarrow x_{i} \in \Omega(f)$, and $x_{i}$ cannot be isolated in $\omega$, for then we would have a contradiction to Lemma 4.2 in section 4 ; just let $F=\omega \backslash\left\{x_{i}\right\}$. Thus, there is an $\epsilon>0$ so that either $\left(x_{i-1}+\epsilon, x_{i}-\epsilon\right)=A$ or $\left(x_{i}+\epsilon, x_{i+1}-\epsilon\right)=B$ contains two points of $\omega$. This, in turn, implies that there is an $N \in \mathbb{N}$ such that $A$ or $B$ contains two points of $\omega_{n}$ whenever $n>N$. Thus, $A$ or $B$ contains a periodic point $x_{n}$ of $g_{n}$, and this contradicts Lemma 5.3.

Our final two results involve $R(\Omega)$, the range of our function $\Omega$ : $\{C(I, I),\|\cdot\|\} \rightarrow\left\{\mathcal{K}^{*}, \mathcal{H}^{*}\right\}$. While they in no way characterize the elements of $R(\Omega)$, they do indicate that $R(\Omega)$ is a very special subset of $\left\{\mathcal{K}^{*}, \mathcal{H}^{*}\right\}$. In fact, the structure of elements in $R(\Omega)$ can be wildly complicated, as $\{[0,1]\}$ is not in the closure of $R(\Omega)$, but $\mathcal{K}$ is $[3]$.

Theorem 5.2. $R(\Omega)$ is nowhere dense in $\left\{\mathcal{K}^{*}, \mathcal{H}^{*}\right\}$.

Proof. Let $\Omega(f)$ be an element of $R(\Omega)$. Since $\Omega(f)$ is compact, for any $\epsilon>0$ there exists an $\epsilon$-net $K_{1}, K_{2}, \ldots, K_{n}$ of $\Omega(f)$. Moreover, we may take this $\epsilon$-net 
so that each $K_{i}$ is finite yet not a singleton, and $i \neq j$ implies $K_{i} \cap K_{j}=\emptyset$. Suppose $\left\{x_{1}, x_{2}, \ldots, x_{m}\right\}=\cup_{i=1}^{n} K_{i}$, and $i<j$ implies $x_{i}<x_{j}$. Set $\delta=$ $\min \left\{x_{i+1}-x_{i}: i=1,2, \ldots, m-1\right\}$. If $\mathcal{K} \in \mathcal{K}^{*}$ such that $\mathcal{H}^{*}\left(\mathcal{K},\left\{K_{i}\right\}_{i=1}^{n}\right)<\frac{\delta}{2}$, then $\mathcal{K}$ is not an element of $R(\Omega)$, since any element of $R(\Omega)$ must contain a singleton, yet by our choice of $\delta$, if $F \in \mathcal{K}$, then $F$ must contain at least two points.

In fact, we can say a bit more about the structure of $R(\Omega)$ in $\left\{\mathcal{K}^{*}, \mathcal{H}^{*}\right\}$.

Theorem 5.3. $R(\Omega)$ is a porous subset of $\left\{\mathcal{K}^{*}, \mathcal{H}^{*}\right\}$.

Proof. Let $E^{*} \in \mathcal{K}^{*}$, and take $E^{\prime} \in \mathcal{K}^{*}$ so that $E^{\prime}$ is a $\frac{1}{2 n}$-net of $E^{*}$. Say $E^{\prime}=$ $\left\{E_{1}^{\prime}, E_{2}^{\prime}, \ldots, E_{m}^{\prime}\right\}$. Now, let $E=\left\{E_{1}, E_{2}, \ldots, E_{m}\right\}$ be a set of subsets of $P=$ $\left\{0, \frac{1}{3 n}, \frac{2}{3 n}, \frac{1}{n}, \ldots, \frac{3 n-1}{3 n}, 1\right\}$, where $E_{i}=\left\{x \in P:|x-y|<\frac{1}{2 n}, y \in E_{i}^{\prime}\right\}$. Thus, $\mathcal{H}^{*}\left(E^{\prime}, E\right)<\frac{1}{2 n}$, and $E_{i} \in E$ implies that $E_{i}$ cannot be a singleton. Suppose $F \in \mathcal{K}^{*}$ and $\mathcal{H}^{*}(F, E)<\frac{1}{6 n}$. Then $F_{i} \in F$ implies $F_{i}$ is not a singleton, so that $F \notin R(\Omega)$. It follows that $B_{\frac{1}{6 n}}(E)=\left\{F \in \mathcal{K}^{*}: \mathcal{H}^{*}(F, E)<\frac{1}{6 n}\right\}$ has a null intersection with $R(\Omega)$. Since $\mathcal{H}^{*}\left(E^{*}, E\right) \leq \mathcal{H}^{*}\left(E^{*}, E^{\prime}\right)+\mathcal{H}^{*}\left(E^{\prime}, E\right)<\frac{1}{n}$, we see that $B_{\frac{1}{6 n}}(E) \subset B_{\frac{7}{6 n}}\left(E^{*}\right)$ and the porosity of $R(\Omega)$ at $E^{*}$ must be at least $\frac{2}{7}$. Since this holds for any set $E^{*}$ in $\left\{\mathcal{K}^{*}, \mathcal{H}^{*}\right\}$, we conclude that $R(\Omega)$ is porous.

\section{Conclusion}

While we have made some progress in understanding how perturbations in a continuous function affect its iterative behavior, our study of this subject in the context of the maps $\Lambda: C(I, I) \rightarrow \mathcal{K}$ and $\Omega: C(I, I) \rightarrow \mathcal{K}^{*}$ is far from complete.

If we go back to considering the overall continuity structure of our maps $\Lambda$ and $\Omega$, an entire series of problems comes to mind. For example, how can one characterize the points of continuity of $\Omega$ and $\Lambda$ ? What are their Baire classifications? Are they measurable? Problems that are perhaps more tractable, yet still very interesting, can be posed by restricting our attention to subsets $S \subseteq C(I, I)$, such as one parameter families of continuous functions, or those functions that are in some sense nonchaotic or smooth.

Our final result of section 5 - that $R(\Omega)$ is porous, hence nowhere dense, in $\left\{\mathcal{K}^{*}, \mathcal{H}^{*}\right\}$ - begs a series of follow-on queries related to characterizing $R(\Omega)$ in $\mathcal{K}^{*}$. Is $R(\Omega)$ a Borel set? How does $\Omega(f)$ reflect the chaotic properties of $f$, if it does at all? 


\section{References}

[1] L. Block and W. Coppel, Dynamics in one dimension, Lecture Notes in Mathematics, 1513, Springer-Verlag, 1991.

[2] A. Blokh, A. M. Bruckner, P. D. Humke and J. Smital, The space of $\omega$ limit sets of a continuous map of the interval, Trans. Amer. Math. Soc. 348 (1996), 1357-1372.

[3] A. M. Bruckner, Stability in the family of $\omega$-limit sets of continuous self maps of the interval, Real Anal. Ex. 22 (1997), 52-57.

[4] A. M. Bruckner, J. B. Bruckner and B. S. Thomson, Real analysis, Prentice-Hall, 1997.

[5] A. M. Bruckner and J. G. Ceder, Chaos in terms of the map $x \rightarrow \omega(x, f)$, Pac. J. Math. 156 (1992), 63-96.

[6] A. M. Bruckner and J. Smital, The structure of $\omega$-limit sets for continuous maps of the interval, Math. Bohemica 117 (1992), 42-47.

[7] A. M. Bruckner and J. Smital, The structure of $\omega$-limit sets of maps of the interval with zero topological entropy, Ergod. Th. and Dynam. Sys. 13 (1993), 7-19.

[8] R. L. Devaney, Chaotic dynamical systems, Benjamin / Cummings Publ. Co., 1986.

[9] V. Fedorenko, A. Sarkovskii and J. Smital, Characterizations of weakly chaotic maps of the interval, Proc. Amer. Math. Soc. 110 (1990), 141148 .

[10] J. Smital, Chaotic functions with zero topological entropy, Trans. Amer. Math. Soc. 297 (1986), 269-282. 